\title{
Ley de Okun en México: un análisis de la heterogeneidad estatal, 2004-2018
}

\author{
Eduardo Loría, Susana Rojas y Eduardo Martínez ${ }^{1}$
}

\section{Resumen}

A luz de la ley de Okun (1983), en su versión de tasas de crecimiento, se evaluó la sensibilidad del desempleo a la actividad económica estatal en México (desde inicios de 2004 hasta el primer semestre de 2018). Para capturar la heterogeneidad de las 32 entidades federativas se contrastaron los resultados de regresiones individuales con la estimación de datos de panel de efectos fijos. El coeficiente de Okun es significativo y con el signo correcto en 22 entidades federativas, con una variación entre $-7,21$ y $-1,25$. Las diez entidades restantes (con signo incorrecto y coeficiente no significativo) se caracterizan por deficientes desempeños económicos, sociales e institucionales. Se observa que la gran recesión generó cambios estructurales en 15 de las 22 entidades federativas donde sí se cumple la ley de Okun en todo el período; además, en estas 22 entidades el índice del Estado de derecho y la tasa de condiciones críticas de ocupación determinan la sensibilidad del crecimiento del desempleo al crecimiento del producto.

\section{Palabras clave}

Crecimiento económico, desempleo, CEPAL, desarrollo regional, desarrollo local, mercado de trabajo, creación de empleos, ajuste estructural, modelos econométricos, México

\section{Clasificación JEL}

$$
\text { C21, C22, E24 }
$$

\section{Autores}

Eduardo Loría es profesor titular de la Facultad de Economía, fundador y coordinador del Centro de Modelística y Pronósticos Económicos (CEMPE) de la Facultad de Economía de la Universidad Nacional Autónoma de México (UNAM), investigador nacional III del Sistema Nacional de Investigadores y miembro de la Academia Mexicana de Ciencias. Correo electrónico: eduardol@unam.mx.

Susana Rojas es colaboradora en el Centro de Modelística y Pronósticos Económicos (CEMPE) de la Facultad de Economía de la Universidad Nacional Autónoma de México (UNAM), especialista en econometría aplicada por esa Facultad. Correo electrónico: surojasmanzo@gmail.com.

Eduardo Martínez es colaborador en el Centro de Modelística y Pronósticos Económicos (CEMPE) de la Facultad de Economía de la Universidad Nacional Autónoma de México (UNAM) y pasante en la Licenciatura en Economía de esa Facultad. Correo electrónico: I.aleve@hotmail.com.

\footnotetext{
Este artículo es parte del proyecto de investigación "Política monetaria y precarización del mercado laboral en México, una explicación alternativa al lento crecimiento 2000-2020" (IN300218), de la Dirección General de Asuntos del Personal Académico (DGAPA) de la Universidad Nacional Autónoma de México (UNAM), que ejecuta el Centro de Modelística y Pronósticos Económicos (CEMPE) de la Facultad de Economía de esa universidad.
} 


\section{Introducción}

Desde que Okun publicó su artículo fundacional sobre la medición de los efectos macroeconómicos bidireccionales entre la producción y el desempleo (1983, publicado originalmente en 1962), la literatura en general se ha concentrado en la realización de estimaciones con distintas particularidades, entre ellas por países individuales y para períodos específicos, con distintas técnicas econométricas y con formas funcionales ad hoc. En algunos textos, como el libro de macroeconomía de Blanchard, Amighini y Giavazzi (2012), se estima la relación de Okun para países desarrollados en forma individual y para distintos períodos. En este sentido, ha prevalecido la visión macroeconómica de esta importante ley.

Los estudios aplicados que estiman la relación de Okun para países particulares en su versión macroeconómica resultan cruciales para evaluar la calidad del crecimiento económico en términos de su efecto sobre la tasa de desempleo. No obstante, dejan de lado las diferencias de productividad laboral entre las regiones que constituyen a esos países, de acuerdo con la definición de heterogeneidad estructural de la Comisión Económica para América Latina y el Caribe (CEPAL) (Pinto, 1973; Cimoli y Porcile, 2011).

En CEPAL (2016) se plantea que la productividad laboral está asociada positivamente con el crecimiento económico y negativamente con la informalidad, por lo que el grado de heterogeneidad estructural refleja distintas trayectorias de crecimiento y generación de empleos entre regiones de un mismo país. En este sentido, nuestro trabajo está enfocado en la definición cepalina de heterogeneidad estructural entre regiones en México.

De esta manera, al aceptar la hipótesis de la heterogeneidad estructural es plausible considerar que habrá diferentes magnitudes del coeficiente de Okun en las distintas regiones de un país; es decir, distintos grados de respuesta del desempleo ante variaciones del producto. Las asimetrías del coeficiente de Okun entre regiones reflejan aspectos estructurales de desarrollo y crecimiento que caracterizan a sus mercados laborales, como la flexibilidad, la segmentación (Dell'Anno y Solomon, 2008; Islas y Cortez, 2018), la institucionalidad (CEPAL, 2012) y la productividad (Friedman y Wachter, 1974).

En virtud de ello, han aparecido - en varios países y en años recientes - trabajos que estiman la ley de Okun a nivel internacional y subnacional. Por ejemplo, Huang y Yeh (2013) estiman un coeficiente de Okun mayor en países miembros de la Organización de Cooperación y Desarrollo Económicos (OCDE). Ball y otros (2016) encuentran el mismo resultado - un mayor coeficiente de Okun- en países desarrollados en comparación con países en desarrollo.

A nivel subnacional, Melguizo (2015) encuentra que el nivel de actividad económica en las provincias de España desempeña un papel fundamental en la estimación del coeficiente de Okun. Guisinger y otros (2018) estiman diferentes coeficientes de Okun en las regiones de los Estados Unidos, que responden significativamente a una menor sindicalización, mayor nivel educativo y mayor tasa de participación de las mujeres. En el caso de México, solo se encuentra el trabajo de Alarcón y Soto (2017), en que se estima el coeficiente de Okun por entidades federativas sin profundizar en sus determinantes.

En concordancia con CEPAL (2016), aceptamos la hipótesis de la heterogeneidad estructural en México sobre la base de la existencia de brechas estructurales en materia económica, social e institucional entre las 32 entidades federativas. Debido a ello, existen distintas trayectorias de productividad laboral, de crecimiento económico y de generación de empleos que, finalmente, arrojan distintos coeficientes de Okun.

Consideramos que incorporar variables económicas, sociales e institucionales que generan la heterogeneidad estructural es una primera contribución de este trabajo. El primer grupo de variables explica el grado de actividad económica medido a través del crecimiento del PIB, el nivel de PIB per cápita, la tasa de desempleo y las exportaciones totales. En cuanto a las variables sociales, se incluyeron la tasa de condiciones críticas de ocupación (TCCO) y la incidencia delictiva (medida a través de las 
tasas de homicidios y secuestros). Por último, se incorporó el factor institucional, expresado a través del índice del Estado de derecho de 2018. De este modo, la presente investigación se apega a la definición cepalina de brechas estructurales a nivel subnacional; en este caso, entre las 32 entidades federativas que integran a México.

La segunda contribución de nuestro trabajo consiste en que estimamos la ley de Okun para México, en su versión de tasas de crecimiento para el período comprendido entre inicios de 2004 y el segundo trimestre de 2018, que es aquel del que existen las series más largas e integradas de una misma fuente de información (INEGl, 2018a y 2018b). La amplitud del período y la frecuencia de los datos brindaron los grados de libertad suficientes para hacer estimaciones individuales robustas y con sentido económico, que más tarde contrastamos con las estimaciones de datos de panel con efectos fijos y con los resultados de Rodríguez y Peredo (2007), quienes utilizaron la misma especificación a nivel nacional, pero para un período mucho más acotado, del tercer trimestre de 1988 al tercer trimestre de 2003.

Además, probamos que la gran recesión del período 2008-2009 provocó cambios notables en la sensibilidad del desempleo a la actividad económica en 15 de las 22 entidades federativas en que sí opera la ley de Okun, grupo al que llamamos G1. Se observó que en una importante cantidad de entidades federativas dejó de operar la ley de Okun, de modo que habría que buscar otros fundamentos teóricos y empíricos que explicaran la dinámica del desempleo en adelante.

La última contribución consiste en demostrar que la operación de la ley de Okun depende del índice del Estado de derecho y de la tasa de condiciones críticas de ocupación. Esto solo puede probarse en el grupo de 22 entidades federativas en que sí se cumple la ley de Okun (G1). Para las 10 entidades federativas restantes (G2), contrariamente y de manera preocupante, no se encontró significancia del coeficiente de Okun con ninguna de las variables analizadas.

Estos resultados plantean que la instrumentación de las políticas estatales no solo debe considerar factores económicos - sin duda, muy importantes -, sino también, y con mayor razón, factores sociales e institucionales para que los mercados laborales reaccionen a las variaciones de la actividad económica local y nacional.

Las contribuciones anteriores fueron resultado de la siguiente estrategia. Primero se estimaron las relaciones individuales de la ley de Okun para las 32 entidades federativas de México y de ello se destacó un resultado muy relevante: se encontró significancia estadística y signos adecuados solo para 22 entidades. Esto permitió hacer un agrupamiento de 22 entidades federativas (G1), por una parte, y 10 entidades federativas (G2), por otra. Se complementó el análisis individual con estimaciones de datos de panel con efectos fijos, que toman en cuenta la heterogeneidad de los estados así agrupados (Greene, 2003). Los resultados derivados de las estimaciones individuales se respaldan y son congruentes con los obtenidos de las regresiones de panel. A partir del análisis de un amplio número de variables concluimos que G1 destaca sobre G2 en el sentido de que presenta mejor desempeño económico, social e institucional, además de que el índice del Estado de derecho y la tasa de condiciones críticas de ocupación determinan el coeficiente de Okun.

Lo anterior permite señalar que el elevado empleo precario y la deficiencia institucional presentes en G2 mantienen un círculo vicioso de alta informalidad, baja productividad y bajo crecimiento económico, todo lo cual explica la no significancia de la ley de Okun, por lo que se genera una condición de pobreza autorreproducible.

El presente trabajo se divide en seis secciones, además de la introducción. En la segunda sección se presenta una revisión de la ley de Okun en los trabajos aplicados que consideran aspectos estructurales. En la tercera sección se definen las brechas estructurales que están detrás de la hipótesis de heterogeneidad estructural en México y en la cuarta se analizan sus desempeños en relación con la conformación de los dos grupos de análisis. En la quinta sección se vincula el análisis econométrico de series de tiempo con el de datos de panel. En la sexta sección se analizan y discuten los resultados principales. Por último, en la séptima sección se presentan las conclusiones del trabajo. 


\section{Revisión de literatura}

Okun (1983) planteó que existe un círculo dinámico - que se retroalimenta - entre alta desocupación, remuneraciones bajas y bajos beneficios, lo que mantiene una limitada inversión en equipos, viviendas, educación e investigación. En la medida en que el proceso se autorreproduzca en el largo plazo, el costo será muy alto en términos económicos (retraso del producto potencial) y sociales (mayor desempleo).

Por la trascendencia teórica y de política económica que tiene esta relación, el análisis macroeconómico moderno frecuentemente utiliza este enfoque para comprobar una de las regularidades más relevantes de la macroeconomía entre la dinámica - bidireccional- del desempleo y de la producción. Es por eso que a esta relación se le ha atribuido el estatuto de ley macroeconómica. Al respecto, Tobin (1980) comenta que la ley de Okun es una de las regularidades empíricas más confiables de la macroeconomía.

No obstante, casi 60 años después de la publicación de ese artículo fundacional no existe un consenso global sobre la tasa de sensibilidad del desempleo al producto que sea óptima para una economía desarrollada o emergente (Islas y Cortez, 2013), mucho menos si se consideran las diferencias económicas, sociales e institucionales que generan la heterogeneidad estructural entre regiones.

En este sentido, Ball y otros (2016) encuentran que el desempleo es más sensible a la actividad económica - presenta un mayor coeficiente de Okun - cuando el PIB per cápita y la participación del sector terciario en la economía son mayores. Por lo tanto, podría decirse que el grado de desarrollo económico y tecnológico es un determinante crucial de la significancia y de la magnitud del coeficiente de Okun.

Dell'Anno y Solomon (2008) y Guisinger y otros (2018) aportan información concluyente sobre los determinantes del coeficiente de Okun en términos de la composición del mercado de trabajo. En este sentido, plantean que una mayor oferta de trabajo en la economía informal y una menor tasa de participación de la mujer se reflejan en un menor coeficiente de Okun. En el caso extremo, Agénor y Aizenman (1999) plantean que el desplazamiento de los trabajadores calificados y no calificados del sector formal al informal anula la relación entre desempleo y producto en el corto plazo.

Dell'Anno y Solomon (2008) señalan que la relación entre la informalidad y la magnitud del coeficiente de Okun se asocia con la deficiencia institucional, que es un factor crucial que determina la heterogeneidad estructural (Cimoli y Porcile, 2011; CEPAL, 2012); sin embargo, no establecen una relación econométrica entre el coeficiente de Okun y el grado de institucionalidad. Su investigación solo señala que la falta de confianza en instituciones deficientes genera una conducta negativa de los trabajadores porque optan por la informalización ante la política fiscal impositiva. En la medida en que aumenten los empleos en el sector informal, que es el de mayor rezago tecnológico, la productividad laboral agregada será menor, aunque el desempleo abierto sea más bajo. Finalmente, habrá un efecto perjudicial sobre la efectividad del crecimiento económico para reducir el desempleo, es decir, un coeficiente de Okun menor.

En el caso particular de México, Islas y Cortez (2018) sugieren que una mayor informalidad podría reflejarse en un menor coeficiente de Okun. Esto se traduce en que en épocas de recesión el desempleo reaccione de manera más pronunciada a la actividad económica que cuando se está en la fase expansiva del ciclo. Lo mismo ocurre en el caso de la economía estadounidense (Crespo, 2003).

Islas y Cortez (2018), a partir del trabajo de Jardin y Stephan (2012), plantean que la no linealidad del coeficiente de Okun en México se debe a que en períodos de recesión, en la medida en que el mercado laboral sea flexible, las empresas despiden a sus empleados rápida y agresivamente, y no contratan de la misma forma en períodos de expansión. Esto redunda en que el desempleo se reduzca lentamente en el período de recuperación, por lo que los trabajadores desempleados encuentran una salida en la informalidad (Loría, Aupart y Salas, 2016), sobre todo las mujeres (Loría, Libreros y Salas, 2011). 
Los trabajos sobre la ley de Okun en México no se limitan a una relectura del artículo original (Loría y Ramos, 2007), sino que también se han centrado en atribuir la magnitud del coeficiente de Okun a factores como la flexibilidad laboral (Chavarín, 2001; Loría, Ramírez y Salas, 2015), el género (Loría, Libreros y Salas, 2011), la informalidad (Islas y Cortez, 2018) y la fase del ciclo económico (Islas y Cortez, 2013 y 2018). Sin embargo, estos trabajos, aun cuando son meritorios por cuanto incorporan variables que enriquecen el análisis original de Okun (1983), no consideran explícitamente la heterogeneidad estructural que existe en las 32 entidades federativas ni los factores que la generan.

El trabajo de Alarcón y Soto (2017) es el único, entre los que se encontraron disponibles, que estima la ley de Okun para las 32 entidades federativas de México. Los resultados de su investigación se sustentan en técnicas de datos de panel con efectos fijos para pocos datos anuales (2003-2014), por lo que al hacer las estimaciones individuales los autores se enfrentan con resultados poco robustos. Además, no desarrollan ni analizan específicamente las diferencias económicas, sociales o institucionales que generan la heterogeneidad estructural. En esto último reside la contribución del presente trabajo. Reconocemos, entonces, que las limitaciones de los trabajos sobre la ley de Okun aplicados al caso de México motivan la realización de la presente investigación.

\section{Brechas estructurales en México}

En CEPAL (2016) se señala que existen diferencias en materia económica, social e institucional entre las 32 entidades federativas de México, a las que se llama brechas estructurales. Estas brechas que se observan entre regiones (entre sectores productivos, entre empresas orientadas a la exportación y empresas que se concentran en el mercado local, entre empresas de distintos tamaños, entre los distintos grados de calificación y el sexo de los trabajadores y entre la formalidad y la informalidad) causan asimetrías de la productividad laboral y definen la heterogeneidad estructural. Los resultados del trabajo indican que el lento crecimiento está asociado con baja productividad y, a su vez, relacionan esta baja productividad con la existencia de estas brechas estructurales.

En el mismo documento se reconoce que la heterogeneidad entre sectores y regiones, así como el importante peso de las actividades de baja productividad en la economía mexicana, actúan como un obstáculo para el aumento de la productividad laboral en el agregado. Se expone que el sector manufacturero, concentrado en el norte del país, constituye la actividad con mayor crecimiento y dinamismo, que entre 1990 y 2012 presentó el mayor crecimiento (2,1\%), impulsado por las industrias intensivas en escala (que incluyen a la industria automotriz). Como un resultado contrario al anterior, se indica que el menor desempeño lo registraron el sector primario y los estados vinculados a actividades petroleras.

En dicha investigación se identifica una disminución considerable de las horas trabajadas en el sector manufacturero en favor de incrementos en las del sector de los servicios, particularmente del comercio. Esto se traduce en desplazamientos de mano de obra desde sectores de alto crecimiento de la productividad hacia sectores donde la productividad laboral es menos dinámica, con lo que se acentúa la heterogeneidad estructural.

En CEPAL (2016) se concluye que, en general, la mayoría de los estados del norte y del centro del país exhiben una dinámica de productividad laboral mayor que la de los estados del sur. Los estados que mantienen el mayor ritmo de productividad laboral, antes y después de la gran recesión, son Querétaro, Aguascalientes, Zacatecas y Nuevo León, mientras que los estados que registraron el menor crecimiento de la productividad laboral son Campeche y Baja California.

Además, resalta el hecho de que la productividad laboral en México está correlacionada negativamente con la informalidad y positivamente con el crecimiento del PIB y las exportaciones. 
En este sentido, en el presente trabajo también probamos que las entidades federativas que exhiben mayor crecimiento de la productividad laboral son aquellas que registran mayor crecimiento económico, menor informalidad y mayores exportaciones.

Es importante remarcar que las condiciones del mercado laboral, como su composición (trabajo informal frente a trabajo formal y tasa de participación de la mujer), precarización e institucionalidad, reflejan la heterogeneidad estructural del país (CEPAL, 2012). En este sentido, la población desempleada busca la supervivencia ofreciendo su trabajo en empleos precarios y de baja productividad laboral, que están asociados a las microempresas y al sector informal. En presencia de malos diseños de las instituciones del mercado laboral, la precariedad de estos empleos será persistente.

Para avanzar hacia una dinámica de la productividad laboral más homogénea se requiere contar con un Estado activo que diseñe una estrategia sistémica de largo plazo en los ámbitos de las políticas industrial, laboral, social y ambiental; sobre todo, que apoye la participación activa del sector privado en el marco de alianzas y pactos para el desarrollo.

En CEPAL (2012) se enfatiza que las instituciones que fomentan la innovación y el aprendizaje generan efectos virtuosos sobre el crecimiento y la productividad. Sin embargo, cuando fomentan actividades improductivas, como la malversación de recursos, los incentivos para invertir en la economía decaen (Mauro, 1995), al igual que la confianza de la población en esas instituciones (Dell'Anno y Solomon, 2008). Consecuentemente, se reduce la generación de empleos y aquellos individuos que ya están empleados en puestos formales tienen incentivos para migrar al sector informal. En este sentido, existe una relación muy estrecha entre la productividad y el crecimiento económico, variables que a su vez guardan relación con la precarización laboral y las variables institucionales.

Nuestro trabajo coincide con lo planteado en CEPAL (2012 y 2016) en el sentido de que detectamos que la significancia y la magnitud del coeficiente de Okun dependen de las brechas estructurales, que derivan en desempeños heterogéneos de la productividad laboral. Al respecto, es importante mencionar que no existen series de datos estatales que capturen todas las brechas estructurales para un período largo y que sean contrastables con las cifras de empleo y producto.

Aun así, nuestro análisis revela que el coeficiente de Okun no es estadísticamente significativo en los estados en que el crecimiento de la productividad laboral fue bajo, como Morelos, Guerrero y Campeche. Por otro lado, entidades federativas como Querétaro, Ciudad de México y Nuevo León, que muestran altas tasas de crecimiento de la productividad laboral desde 2008, presentan coeficientes de Okun estadísticamente significativos y con el signo correcto.

\section{Heterogeneidad estructural}

Como ya mencionamos, los trabajos sobre la ley de Okun para México, en general, desatienden el problema de la heterogeneidad estructural al hacer estimaciones a nivel macro con distintas especificaciones, períodos y frecuencias de datos. A partir de los elementos teóricos planteados en los apartados anteriores, se realizaron regresiones individuales para detectar las asimetrías del coeficiente de Okun y con ello establecer una agrupación de las entidades federativas para las que existe información empírica concluyente de la ley de Okun en su versión de tasas de crecimiento.

Una vez realizadas las estimaciones individuales ${ }^{2}$, fue posible identificar con claridad dos grupos amplios de entidades federativas: G1 y G2; es decir, se agruparon, respectivamente, aquellas entidades en que $\beta_{1}$ y $\beta_{2}$ son estadísticamente significativas, tienen los signos adecuados y parámetros económicamente aceptables, y aquellas entidades que no presentan esas características. De este primer ejercicio resultó que G1 se compone de 22 entidades federativas y G2 de las 10 entidades

\footnotetext{
2 Véase la metodología econométrica en la sección siguiente.
} 
federativas restantes. Las entidades federativas que conforman G1 son Aguascalientes, Baja California, Baja California Sur, Chihuahua, Ciudad de México, Coahuila, Colima, Estado de México, Guanajuato, Hidalgo, Jalisco, Michoacán, Nayarit, Nuevo León, Querétaro, Quintana Roo, San Luis Potosí, Sinaloa, Sonora, Tamaulipas, Tlaxcala y Veracruz. Este agrupamiento permitió identificar las características (diferencias) estructurales de ambos grupos.

Un primer acercamiento a la hipótesis de heterogeneidad estructural se presenta en el cuadro 1, donde se puede observar que la agrupación realizada no responde a una clara diferencia del perfil productivo sectorial de las entidades federativas, salvo en el caso del sector terciario, en el que G1 es ligeramente superior a G2 sin Campeche ${ }^{3}$. Sin embargo, llama la atención que la participación del sector secundario en la generación del PIB es exactamente igual en G1, G2 sin Campeche y el total nacional.

\section{Cuadro 1}

México: participación sectorial en el PIB por grupos de entidades federativas, 2016

(En porcentajes)

\begin{tabular}{lcccc}
\hline Sector & Nacional & G1 & G2 & G2 sin Campeche \\
\hline Primario & 3 & 4 & 5 & 6 \\
\hline Secundario & 32 & 32 & 38 & 32 \\
\hline Terciario & 65 & 64 & 57 & 62 \\
\hline Total & 100 & 100 & 100 & 100 \\
\hline
\end{tabular}

Fuente: Elaboración propia, sobre la base de Centro de Estudios de las Finanzas Públicas (CEFP), "Evolución de la actividad productiva nacional y de las entidades federativas 2003-2018", Estudio, № 022/2018, Ciudad de México, 2018.

La pequeña diferencia en la participación de los sectores primario y terciario de G1 y G2 parece determinar la heterogeneidad estructural. En este sentido, existe una predominancia de sectores más tecnológicos y menos agrícolas en G1, por lo que esta situación se refleja en una mayor dinámica de la productividad laboral y, por lo tanto, en un mayor crecimiento económico (Padilla-Pérez y Villarreal, 2015).

Una aproximación más clara a los factores que generan la heterogeneidad estructural se puede obtener del análisis del cuadro 2, que permite ver con claridad que nuestra clasificación (G1 y G2) responde adecuadamente a las diferencias sustanciales de las variables económicas, sociales e institucionales.

En todos los casos, G1 presenta mejores indicadores, salvo en la tasa de desempleo, lo que puede deberse a que en estas entidades federativas existen instituciones que fomentan actividades productivas, por lo que existen menos empleos precarios (CEPAL, 2012). Como consecuencia, aunque el desempleo es mayor, sus mercados de trabajo están integrados en mayor medida por empleo formal, por lo que es plausible considerar que el registro de desempleo abierto de G1 es más confiable debido a que refleja con mayor veracidad el funcionamiento de los mercados laborales.

En cuanto a las variables económicas, hay que destacar que, en particular, G1 registra un menor crecimiento de la tasa de desempleo, así como una mayor vinculación con los mercados internacionales. Esto último puede verse a través del valor medio de las exportaciones ${ }^{4}$, que es sustancialmente superior en G1, en comparación con G2 sin Campeche (244.259 dólares frente a 30.924 dólares). De esta manera, aunque el sector secundario no presenta ninguna diferencia en su contribución productiva, la mayor vinculación con el comercio internacional (cinco veces mayor) y lo que ello implica en términos de competitividad y productividad, y quizá la ligera diferencia en cuanto al sector terciario, junto con su mayor intensidad técnica, explican trayectorias muy distintas de crecimiento y desarrollo.

\footnotetext{
3 Hay que destacar los casos de los estados de Campeche y Tabasco, que al tener perfiles productivos eminentemente petroleros generan altas distorsiones en las variables de interés, en particular el primero, por lo que los análisis posteriores de G2 se hacen con y sin Campeche.

4 De los sectores de manufacturas y minería.
} 


\section{Cuadro 2}

México: variables económicas, sociales e institucionales por grupos de entidades federativas, primer trimestre de 2004 a segundo trimestre de 2018

(En tasas, pesos mexicanos, dólares e índices)

\begin{tabular}{lcccc}
\hline & Nacional & G1 & G2 & G2 sin Campeche \\
\hline Tasa de crecimiento del PIB real $^{\mid}$ & 0,66 & 0,74 & 0,34 & 0,51 \\
\hline PIB per cápita $^{b}$ & 150308 & 135959 & 181874 & 97420 \\
\hline Tasa de crecimiento del PIB per cápita $^{c}$ & 0,26 & 0,36 & 0,05 & 0,23 \\
\hline Tasa de desempleo $^{d}$ & 4,03 & 4,38 & 3,25 & 3,32 \\
\hline Tasa de crecimiento de la tasa de desempleo $^{c}$ & 0,43 & 0,36 & 0,58 & 0,48 \\
\hline Tasa de crecimiento de la productividad del trabajo $^{\mathrm{e}}$ & 0,20 & 0,53 & $-0,18$ & 0,39 \\
\hline Exportaciones totales $^{f}$ & 318 233 & 244259 & 53318 & 30924 \\
\hline Tasa de ocupación en el sector informal $^{g}$ & 26,6 & 25,40 & 29,22 & 29,59 \\
\hline Tasa de crecimiento de la ocupación en el sector informal $^{c}$ & $-0,12$ & $-0,20$ & 0,05 & 0,07 \\
\hline Tasa de condiciones críticas de ocupación $^{h}$ & 12,32 & 12,13 & 18,02 & 18,13 \\
\hline Índice del Estado de derecho (2018) $^{i}$ & 0,3922 & 0,3923 & 0,3920 & 0,3878 \\
\hline Tasa de homicidios $^{j}$ & 3,84 & 3,78 & 3,95 & 4,22 \\
\hline Tasa de secuestros $^{j}$ & 0,24 & 0,22 & 0,27 & 0,29 \\
\hline
\end{tabular}

Fuente: Elaboración propia, sobre la base de Instituto Nacional de Estadística y Geografía (INEGl), Estadísticas históricas de México 2014, Aguascalientes, 2015; INEGl, "México: exportaciones por entidad federativa 2007-2015", 2015 [en línea] http://www3.inegi.org.mx/rnm/index.php/catalog/241/related_materials?idPro=; INEGl, "Encuesta Nacional de Ocupación y Empleo (ENOE), población de 15 años y más de edad”, 2018 [en línea] https://www.inegi.org.mx/ programas/enoe/15ymas/; INEGl, "Indicador trimestral de la actividad económica estatal", 2018 [en línea] https://www. inegi.org.mx/temas/itaee/; Comisión Económica para América Latina y el Caribe (CEPAL), Productividad y brechas estructurales en México (LC/MEX/L.1211), Ciudad de México, 2016; World Justice Project (WJP), Índice de Estado de derecho en México 2018: perspectivas y experiencias en los 32 estados del país, Washington, D.C., 2018; Secretariado Ejecutivo del Sistema Nacional de Seguridad Pública, "Incidencia delictiva", 2021 [en línea] https://www.gob.mx/sesnsp/ acciones-y-programas/incidencia-delictiva-87005?idiom=es.

a Para el PIB real estatal se usó el Indicador Trimestral de la Actividad Económica Estatal (ITAEE), índice de volumen físico base 2013 (INEGl, 2018b). Crecimiento medio para todo el período.

b PIB real en pesos mexicanos de 2013 por cada habitante de la entidad federativa correspondiente (INEGl, 2018b). Promedio aritmético del período.

c Crecimiento medio para todo el período.

a Porcentaje de la población económicamente activa que no trabajó siquiera una hora durante la semana de referencia de la Encuesta Nacional de Ocupación y Empleo (ENOE) pero manifestó su disposición de hacerlo y realizó alguna actividad por obtener empleo (INEGI, 2018a). Promedio aritmético del período.

e Calculada con el índice de productividad laboral media total mediante el índice de volumen físico estatal y el índice de población ocupada por entidad federativa, ambos con base en 2008 (CEPAL, 2016). Crecimiento medio del período 2005-2014.

f Exportaciones de la industria minera y manufacturera (INEGI, 2015a y 2015b). Sumatoria de los promedios por entidad federativa en millones de dólares corrientes del período 2007-2017.

g Proporción de la población ocupada en unidades económicas no agropecuarias operadas sin registros contables y que funcionan a partir de los recursos del hogar o de la persona que encabeza la actividad sin que se constituya como empresa (INEGl, 2018a). Crecimiento medio para todo el período.

h Porcentaje de la población ocupada en alguna de las siguientes situaciones: i) trabaja menos de 35 horas a la semana por razones de mercado; ii) trabaja más de 35 horas con ingresos inferiores al salario mínimo, o iii) labora más de 48 horas con una percepción de hasta dos salarios mínimos (INEGI, 2018a). Promedio aritmético del período.

i Mide la percepción de la sociedad a la adherencia al Estado de derecho. A partir de una encuesta a 25.600 ciudadanos (800 en cada estado) se mide el estado de derecho percibido por los encuestados. La medición del índice utiliza ocho factores: límites al poder gubernamental, corrupción, gobierno abierto, derechos fundamentales, orden y seguridad, cumplimiento regulatorio, justicia civil y justicia penal (WJP, 2018). Los valores cercanos a 1 indican una mayor adhesión el Estado de derecho.

j Número de casos por cada 100.000 habitantes en cada entidad federativa (Secretariado Ejecutivo del Sistema Nacional de Seguridad Pública, 2021).

Todas las variables de G2 actúan de manera perjudicial sobre la relación de Okun en la medida en que los tres grupos de variables presentan malos desempeños, por lo que es posible afirmar que en este grupo existe una trampa de pobreza ${ }^{5}$ que puede condenar a esas entidades federativas a mantener un bajo desarrollo y un muy bajo crecimiento económico de estado estacionario. De ser ese el caso, no es difícil pensar que se generan múltiples externalidades y disfuncionalidades sociales que, a su vez, provocan mayores distorsiones, como marginalidad y criminalidad.

\footnotetext{
5 Véanse mayores detalles sobre el concepto de "trampa de pobreza" en Basu (2003) y Azariadis y Stachurski (2005).
} 


\section{Aspectos econométricos}

La ley de Okun (1983) en su versión original se expresa a través de tres ecuaciones. Sin embargo, en el grueso de la literatura aplicada se han utilizado múltiples variantes, todas con la finalidad de capturar de la mejor manera los efectos de la actividad económica sobre el desempleo.

En este trabajo se realiza una práctica econométrica correcta en la medida en que los resultados estadísticos son robustos para el mayor número de entidades federativas ${ }^{6}$. Encontramos resultados económica y estadísticamente congruentes e importantes únicamente con la especificación de las variables (PIB real y tasa de desempleo) transformadas algebraicamente en sus tasas de crecimiento anualizadas. Una ventaja adicional de esta especificación es que al tratarse de variables estacionarias no se tiene el problema de cointegración y, por lo tanto, de eventual espuriedad.

Para series de tiempo:

$$
\dot{u}_{t}=\beta_{1}+\beta_{2} \dot{Y}_{t}+\varepsilon_{t}
$$

Para datos de panel:

$$
\dot{u}_{i t}=\beta_{1 i}+\beta_{2} \dot{Y}_{i t}+\varepsilon_{i t}
$$

En ambos modelos se trata de series trimestrales para el período comprendido entre el primer trimestre de 2004 y el segundo trimestre de 2018, donde $\dot{u}_{t}$ y $\dot{u}_{i t}$ representan el crecimiento trimestral anualizado de la tasa de desempleo; $\dot{Y}_{t}$ y $\dot{Y}_{i t}$ representan el crecimiento trimestral anualizado del Indicador Trimestral de la Actividad Económica Estatal; $\beta_{1}$ y $\beta_{1 i}$ son los términos constantes que representan la tasa de crecimiento natural del desempleo; $\beta_{2}$ representa el coeficiente de Okun, que mide el grado de sensibilidad del crecimiento del desempleo al crecimiento económico; $\varepsilon_{t}$ y $\varepsilon_{i t}$ son los términos de error que captan los errores idiosincráticos de cada entidad federativa y, en el caso de la ecuación (2), se trata del error compuesto por el efecto individual específico y lo que resta del disturbio; $t$ representa el período de cada variable, en tanto que $i$ se refiere al individuo (entidad federativa).

La principal ventaja de complementar el análisis de la ley de Okun con los métodos de series de tiempo y de datos de panel es que permite considerar las diferencias entre entidades federativas. En particular, el modelo de datos de panel con efectos fijos permite calcular $\beta_{1}$ para cada entidad federativa y obtener una estimación de $\beta_{2}$ a nivel nacional y grupal que considere los efectos individuales de cada entidad, mientras que el modelo de series de tiempo permite estimar el $\beta_{2}$ de cada entidad.

Si bien en este trabajo consideramos que ambas técnicas econométricas son complementarias, y por ello enriquecen el análisis, una estimación por el método de panel puede considerarse más eficiente debido a que brinda más información de los datos, más variabilidad, menos colinealidad entre las variables y más grados de libertad (Klevmarken, 1989; Hsiao, 2014).

Al estimar con la ecuación (1), se utilizó como criterio de selección para conformar G1 que $\beta_{1}$ y $\beta_{2}$ fueran, de manera individual y conjunta, estadísticamente significativos al menos al $10 \%$ de confianza y que $\beta_{2}$ fuera menor que 0 y $\beta_{1}$ mayor que 0 ; es decir, que también tuvieran sentido económico. De esta manera resultó que $\mathrm{G} 1=22$ entidades federativas y $\mathrm{G} 2=10$ entidades federativas.

\footnotetext{
6 Se probaron en total siete diferentes especificaciones de la ley de Okun: i) primeras diferencias; ii) diferencia y brecha del PIB como porcentaje del potencial; iii) diferencia y brecha del PIB como la diferencia entre el PIB real y el potencial en unidades monetarias; iv) brecha del desempleo y brecha del PIB (como porcentaje del potencial); v) brecha del desempleo y crecimiento del PIB; vi) crecimiento del desempleo y brecha del PIB (como porcentaje del potencial), y vii) especificación con las variables en tasas de crecimiento anualizadas, que fue la única con la que se obtuvieron resultados robustos.
} 


\section{Análisis y discusión de resultados}

En el cuadro 3 se muestran exclusivamente los resultados de G1. Los números entre paréntesis corresponden a los t-estadísticos de $\beta_{1}$ y $\beta_{2}$ y, en el caso del F-estadístico, a la significancia (probabilidad) conjunta de los regresores en cada modelo.

Cuadro 3

México: estimación de la ley de Okun en tasas de crecimiento en las entidades federativas de G1, primer trimestre de 2004 a segundo trimestre de 2018

\begin{tabular}{|c|c|c|c|c|}
\hline Entidad federativa & $\beta_{1}$ & $\beta_{2}$ & $R^{2}$ & F-estadístico \\
\hline Aguascalientes & $\begin{array}{c}10,42 \\
(2,73)\end{array}$ & $\begin{array}{c}-1,85 \\
(-3,08)\end{array}$ & 0,145 & $\begin{array}{c}9,49 \\
(0,00)\end{array}$ \\
\hline Baja California & $\begin{array}{c}23,24 \\
(5,19)\end{array}$ & $\begin{array}{c}-7,21 \\
(-8,23)\end{array}$ & 0,563 & $\begin{array}{c}67,74 \\
(0,00)\end{array}$ \\
\hline Baja California Sur & $\begin{array}{c}16,03 \\
(2,93)\end{array}$ & $\begin{array}{l}-1,32 \\
(-2,14)\end{array}$ & 0,076 & $\begin{array}{c}4,60 \\
(0,03)\end{array}$ \\
\hline Chihuahua & $\begin{array}{c}19,44 \\
(3,91)\end{array}$ & $\begin{array}{c}-4,8 \\
(-5,04)\end{array}$ & 0,313 & $\begin{array}{c}25,46 \\
(0,00)\end{array}$ \\
\hline Ciudad de México & $\begin{array}{c}7,88 \\
(3,23)\end{array}$ & $\begin{array}{c}-2,62 \\
(-3,91)\end{array}$ & 0,214 & $\begin{array}{c}15,28 \\
(0,00)\end{array}$ \\
\hline Coahuila & $\begin{array}{c}5,07 \\
(1,91)\end{array}$ & $\begin{array}{c}-1,81 \\
(-5,06)\end{array}$ & 0,314 & $\begin{array}{c}25,66 \\
(0,00)\end{array}$ \\
\hline Colima & $\begin{array}{c}16,23 \\
(3,11)\end{array}$ & $\begin{array}{c}-3,01 \\
(-2,87)\end{array}$ & 0,128 & $\begin{array}{c}8,23 \\
(0,00)\end{array}$ \\
\hline Estado de México & $\begin{array}{c}10,49 \\
(3,89)\end{array}$ & $\begin{array}{c}-3,17 \\
(-5,08)\end{array}$ & 0,316 & $\begin{array}{c}25,82 \\
(0,00)\end{array}$ \\
\hline Guanajuato & $\begin{array}{c}8,42 \\
(2,17)\end{array}$ & $\begin{array}{c}-2,19 \\
(-2,83)\end{array}$ & 0,125 & $\begin{array}{c}8,01 \\
(0,00)\end{array}$ \\
\hline Hidalgo & $\begin{array}{c}21,32 \\
(3,80)\end{array}$ & $\begin{array}{c}-4,83 \\
(-4,13)\end{array}$ & 0,234 & $\begin{array}{c}17,10 \\
(0,00)\end{array}$ \\
\hline Jalisco & $\begin{array}{c}11,37 \\
(3,44)\end{array}$ & $\begin{array}{c}-3,47 \\
(-4,57)\end{array}$ & 0,272 & $\begin{array}{c}20,90 \\
(0,00)\end{array}$ \\
\hline Michoacán & $\begin{array}{c}10,71 \\
(1,87)\end{array}$ & $\begin{array}{c}-2,28 \\
(-1,70)\end{array}$ & 0,049 & $\begin{array}{c}2,90 \\
(0,09)\end{array}$ \\
\hline Nayarit & $\begin{array}{c}12,66 \\
(3,62)\end{array}$ & $\begin{array}{c}-2,05 \\
(-3,59)\end{array}$ & 0,188 & $\begin{array}{c}12,94 \\
(0,00)\end{array}$ \\
\hline Nuevo León & $\begin{array}{c}11,82 \\
(3,78)\end{array}$ & $\begin{array}{c}-3,66 \\
(-5,86)\end{array}$ & 0,380 & $\begin{array}{c}34,37 \\
(0,00)\end{array}$ \\
\hline Querétaro & $\begin{array}{c}26,9 \\
(4,50)\end{array}$ & $\begin{array}{c}-4,96 \\
(-4,93)\end{array}$ & 0,303 & $\begin{array}{c}24,31 \\
(0,00)\end{array}$ \\
\hline Quintana Roo & $\begin{array}{c}18,64 \\
(3,93)\end{array}$ & $\begin{array}{c}-2,71 \\
(-4,16)\end{array}$ & 0,236 & $\begin{array}{c}17,34 \\
(0,00)\end{array}$ \\
\hline San Luis Potosí & $\begin{array}{c}13,41 \\
(2,33)\end{array}$ & $\begin{array}{l}-2,6 \\
(-2,16)\end{array}$ & 0,077 & $\begin{array}{c}4,69 \\
(0,03)\end{array}$ \\
\hline Sinaloa & $\begin{array}{c}8,78 \\
(2,28)\end{array}$ & $\begin{array}{l}-2,20 \\
(-2,78)\end{array}$ & 0,121 & $\begin{array}{c}7,72 \\
(0,00)\end{array}$ \\
\hline Sonora & $\begin{array}{c}7,21 \\
(1,77) \\
\end{array}$ & $\begin{array}{c}-2,12 \\
(-2,75)\end{array}$ & 0,119 & $\begin{array}{l}7,57 \\
(0,00)\end{array}$ \\
\hline Tamaulipas & $\begin{array}{c}3,98^{*} \\
(1,29)\end{array}$ & $\begin{array}{c}-1,71 \\
(-2,43)\end{array}$ & 0,096 & $\begin{array}{c}5,94 \\
(0,01)\end{array}$ \\
\hline Tlaxcala & $\begin{array}{c}4,77 \\
(2,13)\end{array}$ & $\begin{array}{c}-1,25 \\
(-4,09)\end{array}$ & 0,230 & $\begin{array}{c}16,76 \\
(0,00)\end{array}$ \\
\hline Veracruz & $\begin{array}{c}6,16 \\
(2,01)\end{array}$ & $\begin{array}{c}-1,48 \\
(-1,77)\end{array}$ & 0,053 & $\begin{array}{c}3,15 \\
(0,08)\end{array}$ \\
\hline
\end{tabular}

Fuente: Elaboración propia, sobre la base de Instituto Nacional de Estadística y Geografía (INEGI), "Encuesta Nacional de Ocupación y Empleo (ENOE), población de 15 años y más de edad”, 2018 [en línea] https://www.inegi.org.mx/ programas/enoe/15ymas/; INEGl, "Indicador trimestral de la actividad económica estatal", 2018 [en línea] https://www. inegi.org.mx/temas/itaee/.

Nota: Entre paréntesis se muestran los t-estadísticos de $\beta_{1}$ y $\beta_{2}$ y, la probabilidad del F-estadístico. El asterisco (*) indica que el coeficiente no es significativo al $10 \%$ de confianza. 
De este cuadro se derivan los siguientes resultados:

- Las 22 entidades federativas muestran resultados económica y estadísticamente solventes, a pesar de que existen diferencias importantes en la $R^{2}$.

- En este sentido, destacan los bajos valores de Baja California Sur, Michoacán, San Luis Potosí y Veracruz. Sin embargo, la prueba $\mathrm{F}$ no permite aceptar que $\beta_{1}$ y $\beta_{2}$ sean iguales a cero. El caso de Veracruz es el más delicado, por el valor de aceptación al 10\%.

- Destaca de inmediato la gran asimetría de $\beta_{2}$, que varía entre -7,21 (Baja California) y $-1,25$ (Tlaxcala).

- La lectura de $\beta_{1}$ y $\beta_{2}$ permite responder a la interrogante sobre la tasa de crecimiento del producto requerida para que no aumente la tasa de desempleo. De esta suerte, en los casos de Ciudad de México, Coahuila y Nuevo León, por ejemplo, se requiere de un crecimiento económico de alrededor del 3\%, y en Nayarit, Quintana Roo y Baja California Sur de poco más del $6 \%$. Este punto se comprueba al evaluar la relación $\beta_{1} / \beta_{2}$.

- Es plausible considerar que en la medida en que $\left|\beta_{2}\right|$ es mayor, hay instituciones más eficientes, menor empleo precario y, en consecuencia, mayor eficiencia de los mercados laborales en cuanto a que presentan una mayor productividad (CEPAL, 2012).

Por otro lado, la regresión con series de tiempo para el total nacional y el período completo arroja un coeficiente más sensible que el de la mayoría de las entidades federativas de G1; sin embargo, existen graves problemas de especificación, así como no linealidad, de acuerdo con la prueba RESET de Ramsey:

$$
\begin{aligned}
& \dot{u}_{t}=9,46-3,62 * \dot{Y}_{t}+\varepsilon_{t} \\
& t \quad(4,88) \quad(-6,52)
\end{aligned}
$$

$R^{2}=0,43 ; \mathrm{F}=42,51(0,00) ; \mathrm{DW}=0,58 ; \mathrm{JB}=12,19(0,00) ; \mathrm{LM}(4)=10,41(0,00) ; \mathrm{ARCH}(2)=1,18(0,31) ;$ $\mathrm{ARCH}(4)=2,54(0,51) ;$ White(n.c) = 0,62(0,43); White(c) =0,38(0,68); $\operatorname{Reset}(1)=10,69(0,00)$; Reset $(2)=6,96(0,00)^{7}$.

Un segundo ejercicio econométrico - que representa una importante aportación, debido a que no se realiza en los trabajos referenciados - consiste en probar la hipótesis de cambio estructural. Esto se hace solo para G1 con la prueba de Bai y Perron (1998), que permite detectar rompimientos endógenos (véase el cuadro 4).

\section{Cuadro 4}

México: estimación de la ley de Okun en tasas de crecimiento en las 15 entidades federativas de G1 con cambios estructurales, primer trimestre de 2004 a segundo trimestre de 2018

\begin{tabular}{llcccc}
\hline Entidad federativa & Período significativo & $\beta_{1}$ & $\beta_{2}$ & $R^{2}$ & F-estadístico \\
\hline Aguascalientes & 2004 (trim 1)-2010 (trim 1) & 21,9 & $-2,23$ & 0,24 & 11,56 \\
& & $(5,34)$ & $(-3,30)$ & & $(0,00)$ \\
\hline Baja California Sur & 2007 (trim 3)-2009 (trim 4) & 70,3 & $-2,96$ & 0,66 & 16,08 \\
& & $(9,74)$ & $(-4,01)$ & & $(0,00)$ \\
\cline { 2 - 6 } & 2010 (trim 1)-2018 (trim 2) & $2,3^{*}$ & $-0,89$ & 0,14 & 5,63 \\
& & $(0,69)$ & $(-2,37)$ & & $(0,02)$ \\
\hline Chihuahua & 2004 (trim 1)-2009 (trim 3) & 38,8 & $-5,74$ & 0,43 & 16,24 \\
& & $(4,65)$ & $(-4,03)$ & & $(0,00)$ \\
\hline
\end{tabular}

\footnotetext{
7 Estos resultados contrastan con los obtenidos con la misma especificación por Rodríguez y Peredo (2007) para el período comprendido entre el tercer trimestre de 1988 y el tercer trimestre de 2003. Dichos autores estiman un coeficiente de Okun de $-2,47$.
} 
Cuadro 4 (conclusión)

\begin{tabular}{|c|c|c|c|c|c|}
\hline Entidad federativa & Período significativo & $\beta_{1}$ & $\beta_{2}$ & $R^{2}$ & F-estadístico \\
\hline \multirow[t]{2}{*}{ Ciudad de México } & 2004 (trim 1)-2006 (trim 4) & $\begin{array}{c}28,6 \\
(3,80)\end{array}$ & $\begin{array}{c}-5,59 \\
(-3,17)\end{array}$ & 0,50 & $\begin{array}{c}10,06 \\
(0,00)\end{array}$ \\
\hline & 2007 (trim 1)-2018 (trim 2) & $\begin{array}{l}5,3 \\
(2,31)\end{array}$ & $\begin{array}{c}-2,59 \\
(-3,93)\end{array}$ & 0,26 & $\begin{array}{c}15,50 \\
(0,00)\end{array}$ \\
\hline Coahuila & 2004 (trim 1)-2009 (trim 4) & $\begin{array}{l}10,8 \\
(2,30)\end{array}$ & $\begin{array}{c}-2,46 \\
(-4,05)\end{array}$ & 0,42 & $\begin{array}{c}16,44 \\
(0,00)\end{array}$ \\
\hline Estado de México & 2004 (trim 1)-2009 (trim 4) & $\begin{array}{l}16,0 \\
(4,21)\end{array}$ & $\begin{array}{l}-3,9 \\
(-4,19)\end{array}$ & 0,42 & $\begin{array}{c}17,62 \\
(0,00)\end{array}$ \\
\hline Hidalgo & 2004 (trim 1)-2009 (trim 3) & $\begin{array}{l}41,6 \\
(4,36)\end{array}$ & $\begin{array}{c}-6,34 \\
(-3,28)\end{array}$ & 0,33 & $\begin{array}{c}10,79 \\
(0,00)\end{array}$ \\
\hline Jalisco & 2004 (trim 1)-2016 (trim 1) & $\begin{array}{l}14,2 \\
(4,65)\end{array}$ & $\begin{array}{c}-3,40 \\
(-4,88)\end{array}$ & 0,33 & $\begin{array}{c}23,87 \\
(0,00)\end{array}$ \\
\hline \multirow[t]{3}{*}{ Nayarit } & 2004 (trim 1)-2005 (trim 4) & $\begin{array}{l}77,3 \\
(3,87)\end{array}$ & $\begin{array}{c}-7,64 \\
(-4,06)\end{array}$ & 0,73 & $\begin{array}{c}16,50 \\
(0,00)\end{array}$ \\
\hline & 2006 (trim 1)-2015 (trim 2) & $\begin{array}{l}13,4 \\
(5,48)\end{array}$ & $\begin{array}{c}-1,28 \\
(-2,89)\end{array}$ & 0,18 & $\begin{array}{c}8,39 \\
(0,00)\end{array}$ \\
\hline & 2015 (trim 3)-2018 (trim 2) & $\begin{array}{l}-2,1^{*} \\
(-0,44)\end{array}$ & $\begin{array}{c}-3,61 \\
(-2,56)\end{array}$ & 0,39 & $\begin{array}{c}6,58 \\
(0,02) \\
\end{array}$ \\
\hline Nuevo León & 2004 (trim 1)-2010 (trim 1) & $\begin{array}{l}23,5 \\
(4,65)\end{array}$ & $\begin{array}{c}-4,45 \\
(-5,21)\end{array}$ & 0,54 & $\begin{array}{c}27,24 \\
(0,00)\end{array}$ \\
\hline \multirow[t]{2}{*}{ Querétaro } & 2004 (trim 1)-2009 (trim 1) & $\begin{array}{l}28,1 \\
(2,43)\end{array}$ & $\begin{array}{c}-4,47 \\
(-2,29)\end{array}$ & 0,21 & $\begin{array}{c}5,25 \\
(0,03)\end{array}$ \\
\hline & 2009 (trim 2)-2011 (trim 1) & $\begin{array}{l}74,1 \\
(12,45)\end{array}$ & $\begin{array}{c}-12,61 \\
(-12,11)\end{array}$ & 0,96 & $\begin{array}{c}146 \\
(0,00)\end{array}$ \\
\hline Quintana Roo & 2009 (trim 2)-2018 (trim 2) & $\begin{array}{c}20,2 \\
(6,72)\end{array}$ & $\begin{array}{c}-5,01 \\
(-10,15)\end{array}$ & 0,74 & $\begin{array}{r}103,05 \\
(0,00) \\
\end{array}$ \\
\hline \multirow[t]{2}{*}{ Sinaloa } & 2004 (trim 1)-2005 (trim 4) & $\begin{array}{l}43,9 \\
(4,25)\end{array}$ & $\begin{array}{c}-11,95 \\
(-5,08)\end{array}$ & 0,81 & $\begin{array}{c}25,87 \\
(0,00)\end{array}$ \\
\hline & 2006 (trim 1)-2018 (trim 2) & $\begin{array}{l}6,2 \\
(1,67)\end{array}$ & $\begin{array}{c}-1,43 \\
(-1,90) \\
\end{array}$ & 0,07 & $\begin{array}{c}3,64 \\
(0,06) \\
\end{array}$ \\
\hline Tamaulipas & 2004 (trim 1)-2011 (trim 2) & $\begin{array}{l}15,1 \\
(3,12)\end{array}$ & $\begin{array}{c}-2,43 \\
(-2,80)\end{array}$ & 0,21 & $\begin{array}{l}7,85 \\
(0,00)\end{array}$ \\
\hline Tlaxcala & 2004 (trim 1)-2011 (trim 1) & $\begin{array}{l}15,9 \\
(5,59)\end{array}$ & $\begin{array}{c}-1,45 \\
(-4,62) \\
\end{array}$ & 0,44 & $\begin{array}{c}21,42 \\
(0,00)\end{array}$ \\
\hline
\end{tabular}

Fuente: Elaboración propia, sobre la base de Instituto Nacional de Estadística y Geografía (INEGI), "Encuesta Nacional de Ocupación y Empleo (ENOE), población de 15 años y más de edad”, 2018 [en línea] https://www.inegi.org.mx/ programas/enoe/15ymas/; INEGI, "Indicador trimestral de la actividad económica estatal”, 2018 [en línea] https:// www.inegi.org.mx/temas/itaee/.

Nota: Únicamente se registran los períodos en que el coeficiente de Okun es significativo al menos al 10\% de confianza. Entre paréntesis se muestran los estadísticos de $\beta_{1}$ y $\beta_{2}$ y la probabilidad del F-estadístico. El asterisco (*) indica que el coeficiente no es significativo al 10\% de confianza.

A partir de lo anterior se encontraron los siguientes resultados:

- En 15 entidades federativas hay al menos un cambio estructural durante todo el período de estudio, lo que implica que solo en 7 los parámetros son estables.

- Únicamente en 5 entidades federativas de 15, la ley de Okun continúa operando hasta el segundo trimestre de 2018, que es la última observación del análisis. Estas son: Baja California Sur, Ciudad de México, Nayarit, Quintana Roo y Sinaloa. En las 10 entidades restantes esta ley dejó de operar en algún momento después de la gran recesión.

- En las entidades que presentan múltiples cambios estructurales, como Baja California Sur, Ciudad de México, Nayarit y Sinaloa, dicha ley sigue operando, pero con cambios notables en la magnitud de $\beta_{2}$. Llama la atención el caso de Nayarit por el aumento notable del coeficiente de Okun en el último período, mientras que en Baja California Sur, Ciudad de México y Sinaloa dicho coeficiente se redujo.

- En estas cuatro entidades federativas el ajuste para el último período que presenta significancia cae notablemente. 
- Llama la atención, como caso único, Querétaro ${ }^{8}$, que tiene dos regímenes (primer trimestre de 2004 a primer trimestre de 2009 y segundo trimestre de 2009 a primer trimestre de 2011), en el segundo de los cuales el ajuste crece mucho (de 0,21 a 0,96$)^{9}$, pero desde el segundo trimestre de 2011 deja de operar la ley de Okun.

Las pruebas de Bai y Perron (1998) y de Quandt-Andrews (derivada de la prueba de Quandt (1960) y presentada en Andrews (1993)) para el total nacional muestran que existe cambio estructural en el cuarto trimestre de $2011^{10}$, por lo que se hicieron dos regresiones por tramos.

La estimación correspondiente al período comprendido entre el primer trimestre de 2004 y el cuarto trimestre de 2011 indica que el coeficiente de Okun es significativo, con signo correcto y con magnitud similar a la de todo el período. Tiene un mejor ajuste, aunque presenta problemas de autocorrelación serial hasta de orden 4. Por el tipo de modelo, es plausible sugerir que se trata de autocorrelación pura; es decir, que por su construcción teórica incurre en la falta de información sistemática ${ }^{11}$ :

$$
\begin{aligned}
\dot{u}_{t} & =14,49-3,56 * \dot{Y}_{t}+\varepsilon_{t} \\
t & (6,05) \quad(-5,90)
\end{aligned}
$$

$R^{2}=0,53 ; \mathrm{F}=34,82(0,00) ; \mathrm{Wald}=48,05(0,00) ; \mathrm{JB}=2,10(0,34) ; \mathrm{ARCH}(2)=0,28(0,75) ; \mathrm{ARCH}(4)=$ $0,83(0,51)$; White(n.c) =0,13(0,71); White(c) =0,13(0,87); $\operatorname{Reset}(1)=2,68(0,11) ; \operatorname{Reset}(2)=1,77(0,18)$.

La estimación del período comprendido entre el primer trimestre de 2012 y el segundo trimestre de 2018 no tiene significancia estadística, lo que revela nuevamente que, así como ocurrió para varias entidades federativas de G1, también para el total nacional la ley de Okun dejó de operar después de la gran recesión:

$$
\begin{aligned}
& \dot{u}_{t}=1,88-1,72 * \dot{Y}_{t}+\varepsilon_{t} \\
& t \quad(-0,62)(-1,59)
\end{aligned}
$$

$R^{2}=0,09 ; \mathrm{F}=2,54(0,12) ;$ Wald $=2,69(0,11) ; \mathrm{JB}=1,18(0,55) ; \mathrm{ARCH}(2)=0,54(0,58) ; \mathrm{ARCH}(4)=$ 1,43(0,26); White(n.c) = 0,17(0,67); White(c) =0,49(0,61); $\operatorname{Reset}(1)=0,48(0,82) ; \operatorname{Reset}(2)=0,28(0,75)$.

Estos cambios estructurales serían reflejo de profundas alteraciones en los mercados laborales, lo que supondría notorias modificaciones en la conducta de contratación y despido, particularmente en cuanto a la reducción de incentivos para contratar personal. Lo anterior se produce ante la incertidumbre de que la fase de recuperación posterior a la gran recesión pueda no ser permanente o darse con firmeza. Estas modificaciones también podrían deberse a cambios en la relación entre capital y trabajo, así como cambios de la productividad laboral en las economías estatales, por lo que la fase de recuperación ha estado acompañada por un menor arrastre de la ocupación. Esta hipótesis es acorde a la no linealidad de la ley de Okun que documentan Islas y Cortez (2013 y 2018).

Con la finalidad de fortalecer estos resultados, en el cuadro 5 se presentan las pruebas de correcta especificación de los modelos de panel de G1, G2 y el conjunto de las 32 entidades federativas.

\footnotetext{
8 Estado que se destaca a nivel nacional por mostrar los mejores indicadores aquí presentados.

9 De hecho, ningún estado reporta un coeficiente de determinación tan alto.

${ }^{10}$ Maximum LR (razón de verosimilitud máxima), F-estadístico (cuarto trimestre de 2011) = 12,66(0,00), maximum Wald F-estadístico (cuarto trimestre de 2011) $=25,33(0,00)$, Ho = no presenta cambio estructural.

${ }^{11}$ Con la finalidad de estimar un modelo robusto en términos de homocedasticidad y no autocorrelación serial, empleamos el método de Newey-West (que utiliza la matriz de ponderación HAC) (IHS Global, 2019), que blinda los errores estándares contra heterocedasticidad y autocorrelación.
} 


\section{Cuadro 5}

México: pruebas de correcta especificación con datos de panel por grupos, primer trimestre de 2004 a segundo trimestre de 2018

\begin{tabular}{|c|c|c|c|c|c|}
\hline Prueba & Hausman & Pesaran & Wald & Breusch-Pagan & Wooldridge \\
\hline Ho: & Exogeneidad estricta & Errores i id & Errores homocedásticos & $\begin{array}{l}\text { Variaciones de panel } \\
\text { iguales a cero }\end{array}$ & $\begin{array}{l}\text { No autocorrelación } \\
\text { de orden } 1\end{array}$ \\
\hline Nacional & $\begin{aligned} \chi^{2}(1) & =13,21 \\
\text { prob } & =0,00\end{aligned}$ & prob $=0,00$ & $\begin{aligned} \chi^{2}(32) & =854,92 \\
\text { prob } & =0,00\end{aligned}$ & & $\begin{aligned} \text { F-stat } & =57,31 \\
\text { prob } & =0,00\end{aligned}$ \\
\hline Grupo 1 & $\begin{aligned} \chi^{2}(1) & =21,36 \\
\text { prob } & =0,00\end{aligned}$ & prob $=0,00$ & $\begin{aligned} \chi^{2}(22) & =476,22 \\
\text { prob } & =0,00\end{aligned}$ & & $\begin{array}{c}\text { F-stat }=27,886 \\
\text { prob }=0,00\end{array}$ \\
\hline Grupo 2 & $\begin{array}{c}\chi^{2}(1)=0,19 \\
\text { prob }=0,65\end{array}$ & & & $\begin{array}{c}\operatorname{chibar}^{2}(01)=0,02 \\
\operatorname{prob}=0,43\end{array}$ & $\begin{aligned} \text { F-stat } & =36,21 \\
\text { prob } & =0,00\end{aligned}$ \\
\hline
\end{tabular}

Fuente: Elaboración propia, sobre la base de Instituto Nacional de Estadística y Geografía (INEGl), "Encuesta Nacional de Ocupación y Empleo (ENOE), población de 15 años y más de edad”, 2018 [en línea] https://www.inegi.org.mx/ programas/enoe/15ymas/; INEGI, "Indicador trimestral de la actividad económica estatal", 2018 [en línea] https://www. inegi.org.mx/temas/itaee/.

Sobresalen los siguientes resultados puntuales para el conjunto de las 32 entidades federativas y para G1:

- La prueba de Hausman (1978) muestra que existe correlación entre los efectos individuales y las variables explicativas; es decir, que no se cumple con exogeneidad estricta, por lo que es preferible una estimación por el método menos eficiente, pero robusto, de efectos fijos. Esta prueba indica que el desempleo de cada entidad federativa no solo depende del desempeño económico, sino también de otras variables, como las variables sociales e institucionales tales como la tasa de condiciones críticas de ocupación y el índice del Estado de derecho, lo que comprobamos más adelante.

- La prueba de Pesaran (2004) pone de manifiesto la existencia de dependencia en sección cruzada, por lo que la estimación es robusta pero no eficiente y puede presentar sesgos en los errores estándar. Esta prueba muestra que el crecimiento de la actividad económica de una entidad federativa influye en la tasa de desempleo de otras.

- La prueba de Wald (Greene, 2003) muestra que existe heterocedasticidad de los residuos y, por lo tanto, se demuestra que existen cambios estructurales en el período de estudio, algo que es coherente con los resultados que se presentan en el cuadro 4.

- La prueba de correlación serial de Wooldridge (Drukker, 2003) muestra que existe autocorrelación serial de orden 1, por lo que las desviaciones estándar de los coeficientes de la estimación se registran más bajas de lo que en realidad son, además de que la R2 se registra más alta de lo que en realidad es. Esta prueba muestra que el nivel del desempleo actual depende del desempeño pasado de la actividad económica.

Para G2, las pruebas de Hausman (1978) y de Breusch y Pagan (1979) indican que es preferible una estimación agrupada (pooled) al no existir efecto de panel, en virtud de que los efectos individuales de la actividad económica sobre el desempleo no presentan un comportamiento particular. La estimación de panel de G2 también presenta autocorrelación de orden 1.

Sobre la base de las pruebas anteriores se proponen como las estimaciones más eficientes para el conjunto de las 32 entidades federativas y G1 las que se obtienen con el método de efectos fijos con errores estándar robustos y control de correlación serial, y para G2 una estimación agrupada (pooled) con control de correlación serial y entre individuos (Cameron y Trivedi, 2010) (véase el cuadro 6). 


\section{Cuadro 6}

México: estimaciones de panel de la ley de Okun en tasas de crecimiento, primer trimestre de 2004 a segundo trimestre de 2018

\begin{tabular}{|c|c|c|c|c|c|}
\hline Método & Estados & $\beta_{1}$ & $\beta_{2}$ & $R^{2}$ & F-estadístico \\
\hline \multirow[t]{2}{*}{ Efectos fijos } & Nacional & $\begin{array}{l}8,01 \\
(0,0000)\end{array}$ & $\begin{array}{l}-1,34 \\
(0,0000)\end{array}$ & 0,0808 & $\begin{array}{l}60,92 \\
(0,0000)\end{array}$ \\
\hline & G1 & $\begin{array}{l}9,28 \\
(0,0000)\end{array}$ & $\begin{array}{l}-1,70 \\
(0,0000)\end{array}$ & 0,1640 & $\begin{array}{l}89,43 \\
(0,0000)\end{array}$ \\
\hline Estimación agrupada & G2 & $\begin{array}{l}2,27^{*} \\
(0,479)\end{array}$ & $\begin{array}{l}-0,09^{*} \\
(0,707)\end{array}$ & & \\
\hline
\end{tabular}

Fuente: Elaboración propia, sobre la base de Instituto Nacional de Estadística y Geografía (INEGI), "Encuesta Nacional de Ocupación y Empleo (ENOE), población de 15 años y más de edad", 2018 [en línea] https://www.inegi.org.mx/programas/enoe/15ymas/; INEGl, "Indicador trimestral de la actividad económica estatal", 2018 [en línea] https://www.inegi.org.mx/temas/itaee/.

Nota: Entre paréntesis se muestra la probabilidad de $\beta_{1}, \beta_{2}$ y del F-estadístico. El asterisco (*) indica que el coeficiente no es significativo al $10 \%$ de confianza. La naturaleza de la estimación agrupada no permite obtener la $\mathrm{R}^{2}$ ni el F-estadístico.

Las estimaciones del cuadro 6 indican que para el conjunto de las 32 entidades federativas y para G1 la tasa de desempleo se reduce un 1,34\% y un 1,70\% por cada punto porcentual de crecimiento económico, respectivamente; sin embargo, el desempleo crece a una tasa natural (constante $\beta_{1}$ ) del $8,01 \%$ y el $9,28 \%$, respectivamente, en ausencia de otros factores, lo que implica que el crecimiento económico debe ser del $6,0 \%(8,01 / 1,34)$ y del $5,4 \%(9,28 / 1,70)$, respectivamente, para mantener estable la tasa de desempleo en esos grupos de estados.

Por otro lado, como se demostró en el primer ejercicio econométrico, la estimación de la Ley de Okun para G2 por efectos agrupados (pooled) no es significativa; es decir, se confirma que el crecimiento económico no repercute en la evolución de la tasa de desempleo.

Otro método es la estimación de panel con efectos fijos incorporando variables dicotómicas (dummies), que puede ser menos eficiente, pues no controla los problemas de correlación serial, pero permite comparar la dinámica de la tasa de desempleo de las entidades mediante los $\beta_{1}$ estimados.

En este caso, la estimación de panel con efectos fijos para G1 demuestra que existen marcadas diferencias en sus mercados laborales (véase el cuadro 7), ya que se observan diferentes magnitudes de $\beta_{1}$ (tasas naturales de crecimiento del desempleo), mientras que el coeficiente de Okun común a G1 es de $-2,59$.

Los resultados que se muestran en el cuadro 7 son coherentes con las estimaciones individuales presentadas en el cuadro 3, ya que los estados de Hidalgo, Querétaro y Quintana Roo forman parte de las cinco entidades federativas con mayor $\beta_{1}$, mientras que Coahuila, Tamaulipas, Tlaxcala y Veracruz son las entidades con menor $\beta_{1}$, según ambas técnicas econométricas.

Por último, con el propósito de incorporar los determinantes del coeficiente de Okun $\left(\beta_{2}\right)$, se hicieron múltiples regresiones de sección cruzada para G1 y G2 con las variables económicas, sociales e institucionales que se presentaron en el cuadro 2. Sobre esa base, se probó la hipótesis de que el coeficiente de Okun de G1 (variable dependiente) responde al índice de Estado de derecho y a la tasa de condiciones críticas de ocupación de manera significativa y con sentido económico para cada $i$-ésimo estado. No se encontró significancia para el total nacional ni para G2 con ninguna de las variables presentadas en el cuadro 2. 


\section{Cuadro 7}

México: estimación de panel con efectos fijos (variables dicotómicas) para las entidades federativas de G1, primer trimestre de 2004 a segundo trimestre de 2018

\begin{tabular}{|c|c|c|}
\hline \multicolumn{3}{|c|}{$\beta_{2}=-2,59(0,0000)$} \\
\hline Entidad federativa & $\beta_{1}$ & Error estándar \\
\hline Aguascalientes & $\begin{array}{l}13,83 \\
(0,0000)\end{array}$ & 0,74 \\
\hline Baja California & $\begin{array}{c}13,72 \\
(0,0000)\end{array}$ & 0,08 \\
\hline Baja California Sur & $\begin{array}{l}23,3 \\
(0,0000)\end{array}$ & 1,17 \\
\hline Chihuahua & $\begin{array}{l}12,7 \\
(0,0000)\end{array}$ & 1,87 \\
\hline Ciudad de México & $\begin{array}{l}7,84 \\
(0,0000) \\
\end{array}$ & 0,96 \\
\hline Coahuila & $\begin{array}{l}7,09 \\
(0,0000)\end{array}$ & 0,00 \\
\hline Colima & $\begin{array}{c}14,91 \\
(0,0000)\end{array}$ & 0,24 \\
\hline Estado de México & $\begin{array}{c}8,82 \\
(0,0000)\end{array}$ & 0,13 \\
\hline Guanajuato & $\begin{array}{l}9,91 \\
(0,0000)\end{array}$ & 0,40 \\
\hline Hidalgo & $\begin{array}{l}14,98 \\
(0,0000)\end{array}$ & 0,10 \\
\hline Jalisco & $\begin{array}{l}8,69 \\
(0,0000)\end{array}$ & 0,19 \\
\hline Michoacán & $\begin{array}{l}11,57 \\
(0,0000)\end{array}$ & 0,04 \\
\hline Nayarit & $\begin{array}{l}14,56 \\
(0,0000)\end{array}$ & 0,32 \\
\hline Nuevo León & $\begin{array}{c}8,29^{*} \\
(0,1080)\end{array}$ & 0,28 \\
\hline Querétaro & $\begin{array}{l}16,06 \\
(0,0000)\end{array}$ & 0,76 \\
\hline Quintana Roo & $\begin{array}{l}18,1 \\
(0,0000)\end{array}$ & 0,85 \\
\hline San Luis Potosí & $\begin{array}{l}13,42 \\
(0,0000)\end{array}$ & 0,39 \\
\hline Sinaloa & $\begin{array}{l}9,86 \\
(0,0000) \\
\end{array}$ & 0,05 \\
\hline Sonora & $\begin{array}{l}8,77 \\
(0,0004)\end{array}$ & 0,26 \\
\hline Tamaulipas & $\begin{array}{l}5,44 \\
(0,0000) \\
\end{array}$ & 0,34 \\
\hline Tlaxcala & $\begin{array}{l}6,49 \\
(0,0052)\end{array}$ & 0,48 \\
\hline Veracruz & $\begin{array}{l}8,29 \\
(0,0696)\end{array}$ & 0,24 \\
\hline
\end{tabular}

Fuente: Elaboración propia, sobre la base de Instituto Nacional de Estadística y Geografía (INEGI), "Encuesta Nacional de Ocupación y Empleo (ENOE), población de 15 años y más de edad", 2018 [en línea] https://www. inegi.org.mx/programas/enoe/15ymas/; INEGI, "Indicador trimestral de la actividad económica estatal", 2018 [en línea] https://www.inegi.org.mx/ temas/itaee/.

Nota: Entre paréntesis se indica la probabilidad de $\beta_{1}$ y $\beta_{2}$. El asterisco (*) indica que el coeficiente no es significativo al $10 \%$ de confianza. 
Para probar la hipótesis anterior, se utilizó la matriz de covarianzas coherente con homocedasticidad y autocorrelación de Newey-West (HAC) con el objeto de obtener estimadores eficientes libres de heterocedasticidad y autocorrelación serial (IHS Global, 2019):

$$
\begin{array}{ccc}
\mathrm{CO}_{i}= & 0,12 * \mathrm{TCCO}_{i}-10,64 * I E D_{i}+\varepsilon_{i} \\
t & (2,01) \quad(-6,15)
\end{array}
$$

$R^{2}=0,24 ; \mathrm{JB}=3,66(0,15) ;$ entidad federativa $i=1,2,3, \ldots, 22$.

Se demuestra que una mayor adhesión al Estado de derecho vuelve más efectivo el crecimiento económico para reducir la tasa de desempleo; es decir, se traduce en un mayor coeficiente de Okun en valores absolutos. Por otro lado, en la medida en que es mayor el empleo en condiciones precarias, expresado por la tasa de condiciones críticas de ocupación, se deteriora la magnitud del coeficiente de Okun.

\section{Conclusiones y comentarios finales}

La estimación de la ley de Okun generalmente se ha hecho a nivel macroeconómico a partir de diversas especificaciones, formas funcionales y frecuencias de datos temporales. Esto implica no considerar la existencia de heterogeneidad entre países y entre estados o regiones dentro de un país.

Considerando lo anterior, en esta investigación estimamos dicha ley para las 32 entidades federativas de México durante el período más largo y coherente posible (primer trimestre de 2004 a segundo trimestre de 2018). Incorporamos al análisis tres grupos de variables (económicas, sociales e institucionales) que configuran la heterogeneidad estructural que prevalece en ellas (CEPAL, 2016). La incorporación de estas variables nos permite afirmar que la significancia y la magnitud del coeficiente de Okun dependen de ellas.

A partir de la especificación de la ley de Okun en tasas de crecimiento, encontramos los siguientes resultados puntuales:

- Únicamente en 22 entidades federativas (G1) el mercado laboral reacciona significativamente y en sentido correcto al crecimiento de la actividad económica. En estas entidades se ha registrado mejor desempeño económico (en cuanto a crecimiento económico, crecimiento de la productividad laboral, menor crecimiento del desempleo, mayor nivel del PIB per cápita, mayores exportaciones y disminución de la informalidad), social (menores tasas de criminalidad y menor empleo en condiciones precarias) e institucional (mayor índice del Estado de derecho).

- Al estimar la ley de Okun con series de tiempo y con datos de panel con efectos fijos encontramos información concluyente sobre asimetrías del coeficiente de Okun en G1. Esta asimetría refleja distintos grados de respuesta del mercado laboral al crecimiento del PIB, ya que el coeficiente de Okun está en un rango de -7,25 a -1,25.

- En G2, donde no opera la ley de Okun, no se encontró información concluyente de efecto de panel, lo que significa que la varianza debido al efecto individual no es significativa; además, este grupo muestra homogeneidad en términos de muy malos resultados en todas las variables analizadas, pues se observa que existe un deficiente desempeño económico, social e institucional. 
- El coeficiente de Okun no solo difiere entre las entidades federativas de G1, sino que también varía a través del tiempo. La prueba de Bai y Perron (1998) de cambio estructural muestra que el efecto de la gran recesión del período 2008-2009 ha condicionado la magnitud y la significancia del coeficiente de Okun en la mayoría de las entidades de este grupo.

- Estos resultados diferenciados en G1 denotan diferentes niveles de crecimiento económico requeridos para mantener estable la tasa de desempleo y, por lo tanto, demuestran la heterogeneidad en este grupo.

- De todos los intentos por identificar los determinantes de la ley de Okun a partir de las variables económicas, sociales e institucionales, únicamente el índice del Estado de derecho y la tasa de condiciones críticas de ocupación explican de manera significativa y con sentido económico la magnitud y la significancia del coeficiente de Okun en G1. En virtud de lo anterior, puede afirmarse que una mayor adhesión al Estado de derecho y un menor empleo en condiciones precarias vuelven más efectivo el crecimiento económico para disminuir la tasa de desempleo; es decir, se traducen en que sea mayor el coeficiente de Okun (en valores absolutos).

Más allá de las diferentes magnitudes del coeficiente de Okun estimadas por los métodos econométricos aquí utilizados, una conclusión fundamental es que la recuperación de la institucionalidad y la reducción de la precarización laboral, medidas por el índice del Estado de derecho y la tasa de condiciones críticas de ocupación, respectivamente, son cruciales para fortalecer el funcionamiento de los mercados laborales en G1 y, posiblemente, para que el crecimiento del desempleo recupere su sensibilidad al crecimiento del producto en G2. Con ello se lograría elevar la eficiencia del crecimiento económico sobre la disminución del desempleo a nivel nacional y, a través de la ley de Okun, se podrían generar a la vez efectos virtuosos sobre el crecimiento económico y sobre las demás variables aquí analizadas.

Los resultados del trabajo indican, como un elemento crucial, que la instrumentación de las políticas de desarrollo a nivel estatal y municipal no solo debe ser de índole económica, sino que -más importante aún- debe tener una fuerte orientación de carácter social e institucional que atienda el grave problema de heterogeneidad estructural. De esta manera, una conclusión fundamental es que la mejor reforma institucional, que se debe aplicar con urgencia, es fortalecer el Estado de derecho. Esto tendrá que mejorar las condiciones de ocupación en los mercados laborales y, por lo tanto, generaría efectos virtuosos sobre la productividad laboral, el crecimiento económico y el desarrollo.

\section{Bibliografía}

Agénor, P. R. y J. Aizenman (1999), "Macroeconomic adjustment with segmented labor markets", Journal of Development Economics, vol. 58, № 2, Ámsterdam, Elsevier, abril.

Alarcón, M. e I. Soto (2017), "Heterogeneidad estructural en la estimación de la Ley de Okun para el caso mexicano", Realidad, Datos y Espacio: Revista Internacional de Estadística y Geografía, vol. 8, № 3, Aguascalientes, Instituto Nacional de Estadística y Geografía (INEGI).

Andrews, D. (1993), "Tests for parameter instability and structural change with unknown change point", Econometrica: Journal of the Econometric Society, vol. 61, № 4, Hoboken, Wiley, julio.

Azariadis, C. y J. Stachurski (2005), "Poverty traps", Handbook of Economic Growth, vol. 1, Ámsterdam, Elsevier. Bai, J. y P. Perron (1998), "Estimating and testing linear models with multiple structural changes", Econometrica: Journal of the Econometric Society, vol. 66, № 1, Hoboken, Wiley, enero.

Ball, L. y otros (2016), "Does one law fit all? Cross-country evidence on Okun's Law", documento presentado en la conferencia Global Labor Markets, París, 1 y 2 de septiembre.

Basu, K. (2003), Analytical Development Economics: The Less Developed Economy Revisited, Cambridge, The MIT Press. 
Blanchard, O., A. Amighini y F. Giavazzi (2012), Macroeconomía, Madrid, Pearson Educación.

Breusch, T. y A. Pagan (1979), "A simple test for heteroscedasticity and random coefficient variation", Econometrica: Journal of the Econometric Society, vol. 47, № 5, Hoboken, Wiley, septiembre.

Cameron, A. y P. Trivedi (2010), Microeconometrics Using Stata, College Station, Stata Press.

CEFP (Centro de Estudios de las Finanzas Públicas) (2018), "Evolución de la actividad productiva nacional y de las entidades federativas 2003-2018", Estudio, № 022/2018, Ciudad de México, julio.

CEPAL (Comisión Económica para América Latina y el Caribe) (2016), Productividad y brechas estructurales en México (LC/MEX/L.1211), Ciudad de México, mayo.

(2012), Cambio estructural para la igualdad: una visión integrada del desarrollo (LC/G.2525(SES.34/4)), Santiago, agosto.

Chavarín, R. (2001), "El costo del desempleo medido en producto: una revisión empírica de la Ley de Okun para México", El Trimestre Económico, vol. 68, № 270, Ciudad de México, Fondo de Cultura Económica (FCE).

Cimoli, M. y G. Porcile (2011), "Tecnología, heterogeneidad y crecimiento: una caja de herramientas estructuralista", MPRA Paper, № 33801, Munich, Ludwig Maximilian University of Munich.

Crespo, J. (2003), "Okun's law revisited", Oxford Bulletin of Economics and Statistics, vol. 65, № 4, Hoboken, Wiley.

Dell'Anno, R. y O. Solomon (2008), "Shadow economy and unemployment rate in USA: is there a structural relationship? An empirical analysis", Applied Economics, vol. 40, № 19, Milton Park, Taylor \& Francis.

Drukker, D. (2003), "Testing for serial correlation in linear panel-data models", The Stata Journal, vol. 3, № 2, Thousand Oaks, SAGE Publishing.

Friedman, B. y M. Wachter (1974), "Unemployment: Okun's law, labor force, and productivity", The Review of Economics and Statistics, vol. 56, № 2, Cambridge, The MIT Press.

Greene, W. (2003), Econometric Analysis, Londres, Pearson.

Guisinger, A. y otros (2018), "A state-level analysis of Okun's law", Regional Science and Urban Economics, vol. 68, Ámsterdam, Elsevier, enero.

Hausman, J. (1978), "Specification tests in econometrics", Econometrica: Journal of the Econometric Society, vol. 46, № 6, Hoboken, Wiley, noviembre.

Hsiao, C. (2014), Analysis of Panel Data, Cambridge, Cambridge University Press.

Huang, H. C. y C. C. Yeh (2013), "Okun's law in panels of countries and states", Applied Economics, vol. 45, № 2, Milton Park, Taylor \& Francis.

IHS Global (2019), "Generalized method of moments" [en línea] http://www.eviews.com/help/helpintro. html\#page/content/gmmiv-Generalized_Method_of_Moments.html.

INEGI (Instituto Nacional de Estadística y Geografía) (2018a), "Encuesta Nacional de Ocupación y Empleo (ENOE), población de 15 años y más de edad" [en línea] https://www.inegi.org.mx/programas/enoe/15ymas/. (2018b), "Indicador trimestral de la actividad económica estatal" [en línea] https://www.inegi.org.mx/ temas/itaee/. (2015a), Estadísticas históricas de México 2014, Aguascalientes.

_ (2015b), "México: exportaciones por entidad federativa 2007-2015" [en línea] http://www3.inegi.org.mx/ rnm/index.php/catalog/241/related_materials?idPro=.

Islas, A. y W. Cortez (2018), “¿Puede el sector informal afectar a la relación entre desempleo y producción? Un análisis del caso de México", Revista CEPAL, № 126 (LC/PUB.2018/26-P), Santiago, Comisión Económica para América Latina y el Caribe (CEPAL), diciembre.

(2013), "Relaciones dinámicas del producto y el empleo en México: una evaluación de sus componentes permanentes y transitorios", Revista CEPAL, № 111 (LC/G.2597-P), Santiago, Comisión Económica para América Latina y el Caribe (CEPAL), diciembre.

Jardin, M. y G. Stephan, G. (2012), "How Okun's law is non-linear in Europe: a semi-parametric approach", Rennes, Universidad de Rennes, abril.

Klevmarken, N. (1989), "Panel studies: what can we learn from them?" European Economic Review, vol. 33, № 2-3, Ámsterdam, Elsevier, marzo.

Loría, E., M. Aupart y E. Salas (2016), "Informalidad, productividad y crecimiento en México, 2000.Q2-2014. Q4", Ensayos: Revista de Economía, vol. 35, № 2, Monterrey, Universidad Autónoma de Nuevo León (UANL), noviembre.

Loría, E., C. Libreros y E. Salas (2011), "La Ley de Okun en México: una mirada de género, 2000.2 2011.1", Investigación Económica, vol. 71, № 280, Ciudad de México, Universidad Nacional Autónoma de México (UNAM). 
Loría, E., E. Ramírez y E. Salas (2015), "La Ley de Okun y la flexibilidad laboral en México: un análisis de cointegración, 1997Q3-2014Q1”, Contaduría y Administración, vol. 60, №3, Ciudad de México, Universidad Nacional Autónoma de México (UNAM).

Loría, E. y M. Ramos (2007), "La ley de Okun: una relectura para México, 1970-2004", Estudios Económicos, vol. 22, No 1, Ciudad de México, El Colegio de México.

Mauro, P. (1995), "Corruption and growth", The Quarterly Journal of Economics, vol. 110, № 3, Oxford, Oxford University Press, agosto.

Melguizo, C. (2015), "An analysis of the Okun's Law for the Spanish provinces", Document de Treball, № 2015/01, Barcelona, Universidad de Barcelona.

Okun, A. (1983), "Potential GNP: its measurement and significance", Economics for Policymaking: Selected Essays of Arthur M. Okun, Cambridge, The MIT Press.

Padilla-Pérez, R. y F. Villarreal (2015), "Unfinished structural change and sectoral heterogeneity: the case of Mexico", MPRA Paper, № 62947, Munich, Ludwig Maximilian University of Munich, enero.

Pesaran, M. (2004), "General diagnostic tests for cross section dependence in panels", IZA Discussion Paper, № 1240, Bonn, Instituto de Economía Laboral (IZA), agosto.

Pinto, A. (1973), "Heterogeneidad estructural y modelo de desarrollo reciente de la América Latina", Inflación: raíces estructurales, Lecturas del Fondo de Cultura Económica, № 3, Ciudad de México, Fondo de Cultura Económica (FCE).

Quandt, R. E. (1960), "Tests of the hypothesis that a linear regression system obeys two separate regimes", Journal of the American Statistical Association, vol. 55, № 290, junio.

Rodríguez, P. y F. Peredo (2007), "Estimación de la ley de Okun para la economía mexicana", Análisis Económico, vol. 22, № 51, Ciudad de México, Universidad Autónoma Metropolitana (UAM).

Secretariado Ejecutivo del Sistema Nacional de Seguridad Pública (2021), "Incidencia delictiva" [en línea] https://www.gob.mx/sesnsp/acciones-y-programas/incidencia-delictiva-87005?idiom=es.

Tobin, J. (1980), "In memoriam: a tribute to Arthur M. Okun", Washington, D.C., The Brookings Institution.

WJP (World Justice Project) (2018), Índice de Estado de derecho en México 2018: perspectivas y experiencias en los 32 estados del país, Washington, D.C. 